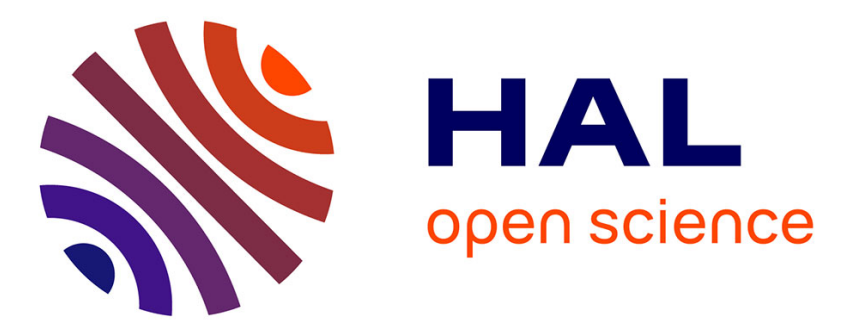

\title{
Local Controllability, Trajectory Planning, and Stabilization of a Two-Agent Underactuated Microrobotic System
}

\author{
Matthieu Fruchard, Lounis Sadelli, Antoine Ferreira
}

\section{- To cite this version:}

Matthieu Fruchard, Lounis Sadelli, Antoine Ferreira. Local Controllability, Trajectory Planning, and Stabilization of a Two-Agent Underactuated Microrobotic System. IEEE Systems Journal, 2020, 14 (2), pp.2892-2900. 10.1109/JSYST.2019.2942780 . hal-03268393

\section{HAL Id: hal-03268393 https://hal.science/hal-03268393}

Submitted on 23 Jun 2021

HAL is a multi-disciplinary open access archive for the deposit and dissemination of scientific research documents, whether they are published or not. The documents may come from teaching and research institutions in France or abroad, or from public or private research centers.
L'archive ouverte pluridisciplinaire HAL, est destinée au dépôt et à la diffusion de documents scientifiques de niveau recherche, publiés ou non, émanant des établissements d'enseignement et de recherche français ou étrangers, des laboratoires publics ou privés. 


\title{
Local Controllability, Trajectory Planning and Stabilization of a Two-Agent Underactuated Microrobotic System
}

\author{
Matthieu Fruchard, Lounis Sadelli, Antoine Ferreira
}

\begin{abstract}
Multi-microagent systems are appealing to perform targeted therapy, biosensing and diagnosis. However on most magnetic platforms, the magnetic field is stationary so all the robots experience the same control input, resulting in an underactuated system. However, to date, the controllability of underactuated microsystems had hardly been addressed. This paper thus investigates the system local controllability. This result highlights the necessity for the agents to operate in close vicinity to achieve trajectory tracking along an admissible reference trajectory whose choice is discussed. We then propose a backstepping controller to locally stabilize the nonlinear system. Simulations illustrate this approach efficiency and limitations for different designs of the microrobotic system.
\end{abstract}

Index Terms-Controllability, Underactuated multi-agent system, Microrobotics, Medical control system synthesis

\section{NOMENCLATURE}

$\eta \quad$ Blood viscosity.

$v_{f} \quad$ Blood velocity.

$\nabla B \quad$ Magnetic field gradient.

$\mu_{0} \quad$ Vacuum permeability.

$\vec{F}_{d} \quad$ Drag force.

$\vec{F}_{m} \quad$ Magnetic motive force.

$\vec{F}_{k j} \quad$ Magnetic interaction exerted by the $k$ th micro-agent on the $j$ th micro-agent.

$\begin{array}{ll}p_{i} & \text { Position of the } i \text { th micro-agent. } \\ r_{i} & \text { Radius of the } i \text { th micro-agent. }\end{array}$

$\rho_{i} \quad$ Density of the $i$ th micro-agent.

$m_{i} \quad$ Mass of the $i$ th micro-agent.

$M_{i} \quad$ Magnetization of the $i$ th micro-agent.

$\tau_{m_{i}} \quad$ Magnetic ratio of the $i$ th micro-agent.

$\mathcal{M}_{i} \quad$ Magnetic moment of the $i$ th micro-agent.

$\beta_{i} \quad$ Wall effect ratio for the $i$ th micro-agent.

a Normalized drag parameter.

$\alpha \quad$ Normalized magnetic sensitivity.

$e \quad$ Normalized magnetic interaction sensitivity.

\section{INTRODUCTION}

Tetherless nano or microrobots control in the circulatory system is a promising challenge for minimally invasive therapy or in situ diagnosis. Most of such systems designs rely on deported magnetic actuation [1], [2], [3], [4] to facilitate miniaturization and increase the payload ratio. Prior works

M. Fruchard is with the Univ. Orléans, Insa-Cvl, Laboratory PRISME, EA 4229, F45072, Orléans, France. matthieu.fruchard@univ-orleans.fr

L. Sadelli was with the Univ. Orléans, Insa-Cvl, Laboratory PRISME, EA 4229, F45072, Orléans, France. He is now with ACTOAT SA, 213 rue Pierre Marti, 25460 Etupes, France. lounis . sadellidext.mpsa . com

A. Ferreira is with the Insa-Cvl, Univ. Orléans, Laboratory PRISME, EA 4229, F18020, Bourges, France. antoine.ferreira@insa-cvl.fr have mainly studied their propulsion feasibility, using either elastic flagellum [5], [6], helical flagellum [7], [8], or bead pulled robots [9], [1], see [10] for a review. Controllability [11] and observability [12] for a single agent have been addressed quite recently. Trajectory planning minimizing the control effort has been defined e.g. in [13], then [14], [15] and [16] have proposed nonlinear controllers and observerbased controllers providing respectively feedback and output feedback Lyapunov stabilization of a single microrobot along a reference trajectory. Yet a single agent can not convey enough payload nor implants, for instance, to be efficient for a therapy [17]: medical applications call for a devoted multi-microagent framework.

\section{A. Multi-Microagent Systems}

Many works have addressed multi-agent strategies and control, yet magnetically actuated microagents are often propelled using the same control input ${ }^{1}$, resulting in a nonlinear underactuated system whose constraints are not compatible with the previously developed approaches. The controllability analysis of such a multi-agent microrobotic system is not trivial [19], [20] and has not been addressed despite its impact on the robots designs. Most of dedicated works rather insist on the independent control of the agents [21], [22], yet the non-integrable constraint precludes independent control. Besides, underactuated systems can not follow any trajectory. Differential flatness has been widely used for such purpose, yet a multi-agent microrobotic system is not generically flat. When the microrobots are far enough from each other, their magnetic interaction forces can be neglected. In such a case, [3], [23], [24] have exploited the difference of step-out frequencies of the robots to select and actuate them separately with a point to point control objective. To the contrary, when the microrobots operate in close vicinity, these magnetic interaction forces are no more negligible. For instance, using a rotating magnetic field actuator, [25] has proposed a bang-bang controller exploiting the magnetic interaction to control the distance and orientation between two microrobots. A more formal control synthesis using a nonlinear model predictive controller has been addressed in the case of either two interaction free microrobots [26], or two microrobots in magnetic interaction [21]. In the former case, the authors have assumed the existence of a solution to the initial optimal control objective, i.e. that the system is locally controllable from the initial to the final point. Yet they did not focused on controllability issues. However, since the robots are far enough to prevent their magnetic interaction, the system is not controllable but simply stable, as proven in the present paper; hence the interest in exploiting

${ }^{1}$ Except the approach developped by [18] using a magnetic coils array. 
the magnetic interaction, as in [21], [25], to prove the system controllability and exploit it to generate admissible trajectories and stabilizing control laws.

\section{B. Contribution}

This paper addresses the local controllability, trajectory planning and stabilization, along admissible trajectories, of a pair of microrobots driven by the same control input. The contribution of this paper is threefold: $i$ ) we first provide a local controllability result, which was -to our best knowledgelacking in the literature, thus giving sufficient conditions for expecting trajectory stabilization; ii) we then propose admissible trajectories for this underactuated system; iii) a Linear Time-Varying (LTV) controller is synthesized to locally stabilize the nonlinear system along any admissible trajectory.

\section{Paper Organization}

This paper is set out as follows. Section II briefly describes the modeling of such a nonlinear system. The contribution is detailed in the next three sections: the controllability of the underactuated system is addressed in Section III, Section IV is devoted to the trajectory planning, and the Lyapunov stabilizing controller is synthesized in Section V. Simulation results illustrate the stability and limitations of the proposed controller in Section VI. Discussion and conclusion are given in Sections VII and VIII.

\section{MODELING}

The dynamics of two magnetic spherical endovascular microrobots $(j)$ and $(k)$, localized by their respective positions $p_{j}$ and $p_{k}$, are subjected to -at least- the drag force, the motive magnetic force and the magnetic interaction between the robots, depicted in Figure 1. The magnetic field is stationary, like in Magnetic Resonance Imagers (MRI). We make no claim to originality in this section devoted to a recall of the forces balance.

\section{A. Forces}

1) Hydrodynamic Drag Force: A microrobot $(i)$ of radius $r_{i}$ and density $\rho_{i}$ moving at relative velocity $\left(\dot{p}_{i}-v_{f}\right)$ in the blood is affected by the drag force. We assume that the Reynolds number is small so the drag is $\operatorname{linear}^{2}$ (Stokes flow):

$$
\vec{F}_{d_{i}}=-m_{i} a_{i}\left(\dot{p}_{i}-v_{f}\right) \vec{i}, \quad a_{i}=\frac{9 \eta}{2 \beta_{i} \rho_{i} r_{i}^{2}}
$$

where $\beta_{i}$ is a ratio related to the wall effect caused by the partial vessel occlusion by the robot [27], and $\eta, m_{i}$ denote respectively the blood viscosity and the microrobot mass.

2) Magnetic Motive Force: The robots are bead pulled, so that the magnetic forces are given by (see e.g. [13]):

$$
\vec{F}_{m_{i}}=m_{i} \alpha_{i} u \vec{i}, \quad \alpha_{i}=\frac{\tau_{m_{i}} M_{i}}{\rho_{i}}
$$

where the scalar control input $u$ is the magnetic field gradient $\nabla B_{x} ; \vec{M}_{i}$ and $\tau_{m_{i}}$ denote the robot $(i)$ magnetization and magnetic material ratio.

\footnotetext{
${ }^{2}$ Yet there is no obstruction in the present work for considering a nonlinear drag force, as in [14], [12].
}

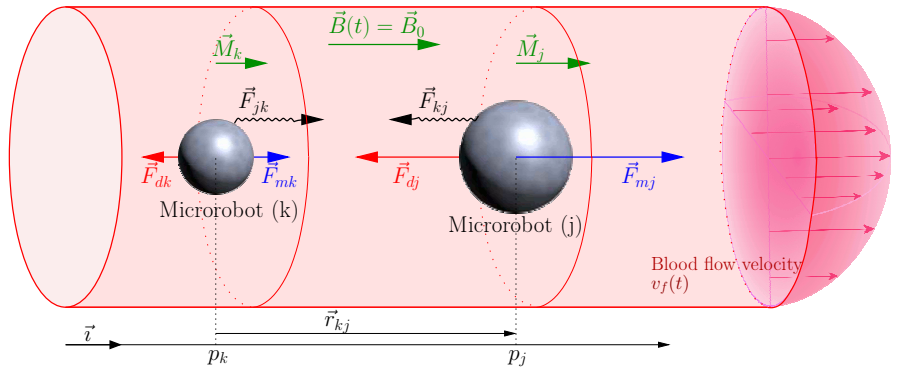

Fig. 1. Forces exerting on two magnetic microrobots $(j)$ and $(k)$ in close vicinity within a blood vessel: $\vec{F}_{d}, \vec{F}_{m}$, and $\vec{F}_{k j}$ respectively denote the drag force, the magnetic motive force, and the magnetic force interaction that the robot $(k)$ exerts on the microrobot $(j)$.

3) Magnetic Interaction Force: Let $\overrightarrow{\mathcal{M}}_{i}=\frac{4}{3} \pi \tau_{m_{i}} r_{i}^{3} \vec{M}_{i}$ denote a microrobot $(i)$ magnetic moment, and $\vec{r}_{j k}=-\vec{r}_{k j}=$ $-\left|p_{k}-p_{j}\right| \vec{\imath}$ as illustrated by Figure 1 . The magnetic interaction exerted by the robot $(k)$ over the robot $(j)$ is given by [28]:

$$
\begin{aligned}
\vec{F}_{k j}= & \frac{3 \mu_{0}\left\|\overrightarrow{\mathcal{M}}_{j}\right\|\left\|\overrightarrow{\mathcal{M}}_{k}\right\|}{4 \pi\left\|\vec{r}_{k j}\right\| 4}\left(\frac{\vec{r}_{k j}\left(\overrightarrow{\mathcal{M}}_{j} \cdot \overrightarrow{\mathcal{M}}_{k}\right)}{\left\|\vec{r}_{k j}\right\|\left\|\overrightarrow{\mathcal{M}}_{j}\right\|\left\|\overrightarrow{\mathcal{M}}_{k}\right\|}+\frac{\overrightarrow{\mathcal{M}}_{j}\left(\vec{r}_{k j} \cdot \overrightarrow{\mathcal{M}}_{k}\right)}{\left\|\overrightarrow{\mathcal{M}}_{j}\right\|\left\|\vec{r}_{k j}\right\|\left\|\overrightarrow{\mathcal{M}}_{k}\right\|}\right. \\
& \left.+\frac{\overrightarrow{\mathcal{M}}_{k}\left(\vec{r}_{k j} \cdot \overrightarrow{\mathcal{M}}_{j}\right)}{\left\|\overrightarrow{\mathcal{M}}_{k}\right\|\left\|\vec{r}_{k j}\right\|\left\|\overrightarrow{\mathcal{M}}_{j}\right\|}-\frac{5 \vec{r}_{k j}}{\left\|\vec{r}_{k j}\right\|} \frac{\left(\vec{r}_{k j} \cdot \overrightarrow{\mathcal{M}}_{j}\right)}{\left\|\vec{r}_{k j}\right\|\left\|\overrightarrow{\mathcal{M}}_{j}\right\|} \frac{\left(\vec{r}_{k j} \cdot \overrightarrow{\mathcal{M}}_{k}\right)}{\left\|\vec{r}_{k j}\right\|\left\|\overrightarrow{\mathcal{M}}_{k}\right\|}\right)
\end{aligned}
$$

Since $\vec{B}$ and $\overrightarrow{\mathcal{M}}_{i}$ are colinear, we obtain:

$$
\vec{F}_{k j}=-\frac{3 \mu_{0} \mathcal{M}_{j} \mathcal{M}_{k}}{2 \pi} \frac{\vec{r}_{k j}}{\left\|\vec{r}_{k j}\right\|^{5}}=-\vec{F}_{j k}
$$

with $\mu_{0}$ the vacuum permeability. This force is a short range attractive interaction between the magnetic robots.

\section{B. State Space Representation}

1) Nonlinear System: Applying the Newton's second law to the microrobots (1) and (2) leads to:

$$
\left\{\begin{array}{l}
m_{1} \ddot{p}_{1} \vec{i}=\vec{F}_{m_{1}}+\vec{F}_{d_{1}}+\vec{F}_{21} \\
m_{2} \ddot{p}_{2} \vec{i}=\vec{F}_{m_{2}}+\vec{F}_{d_{2}}+\vec{F}_{12}
\end{array}\right.
$$

Let $x^{T}=\left[\begin{array}{llll}p_{1} & p_{2} & \dot{p}_{1} & \dot{p}_{2}\end{array}\right]$; using (1), (2), and (4) in (5), we obtain the forthcoming nonlinear dynamics:

$$
\left(\mathcal{S}_{N L}\right):\left\{\begin{array}{l}
\dot{x}_{1}=x_{3} \\
\dot{x}_{2}=x_{4} \\
\dot{x}_{3}=-a_{1} x_{3}-\frac{e_{1}}{\left(x_{1}-x_{2}\right)^{4}}+\alpha_{1} u \\
\dot{x}_{4}=-a_{2} x_{4}+\frac{e_{2}}{\left(x_{1}-x_{2}\right)^{4}}+\alpha_{2} u
\end{array}\right.
$$

with the parameter $e_{i}=\frac{3 \mu_{0} \mathcal{M}_{1} \mathcal{M}_{2}}{2 \pi m_{i}}$.

The system is undefined for $x_{1}=x_{2}$, but even if the contact force has not been modeled here for the sake of simplicity, it is physically not possible for the two robots to share the same position. So, if the first robot initially precedes the second one, then $x_{1}(t)>x_{2}(t), \forall t \geq 0$.

2) LTV System: Along any $\mathcal{C}^{1}$ reference trajectory $x_{r}(t)=$ $\left[x_{r 1}(t), x_{r 2}(t), \dot{x}_{r 1}(t), \dot{x}_{r 2}(t)\right]^{T}$, there exists a $\mathcal{C}^{2}$ gap function $f(t) \in \mathcal{F} \subset \mathbb{R} \backslash\{0\}$ such that $f(t)=x_{r 1}(t)-x_{r 2}(t)$. Linearizing system (6) along such a trajectory leads to the LTV system:

$$
\left(\mathcal{S}_{x}\right): \dot{x}(t)=A_{x}(t) x(t)+B_{x}(t) u(t)
$$


with the time-varying matrices

$$
A_{x}(t) \mid B_{x}=\left[\begin{array}{cccc|c}
0 & 0 & 1 & 0 & 0 \\
0 & 0 & 0 & 1 & 0 \\
\frac{4 e_{1}}{f^{5}(t)} & -\frac{4 e_{1}}{f^{5}(t)} & -a_{1} & 0 & \alpha_{1} \\
-\frac{4 e_{2}}{f^{5}(t)} & \frac{4 e_{2}}{f^{5}(t)} & 0 & -a_{2} & \alpha_{2}
\end{array}\right]
$$

\section{LOCAL CONTROLlABILITY}

We establish a controllability result for the LTV system $\left(\mathcal{S}_{x}\right)$, thus determining sufficient conditions for the nonlinear system controllability. To our best knowledge, this is the first controllability result for an underactuated multi-microagent system. This result impacts both the robots designs and the admissible trajectory issue.

Proposition 1. Let $\chi_{1}=\alpha_{2} e_{1}+\alpha_{1} e_{2}, \chi_{2}=\alpha_{1} \alpha_{2}\left(a_{1}-a_{2}\right)$, $\chi_{3}=\alpha_{1}-\alpha_{2}$ and $\chi_{4}=\alpha_{2} a_{1}-\alpha_{1} a_{2}$. A sufficient condition for the controllability for the nonlinear system $\left(\mathcal{S}_{N L}\right)$ is to satisfy the three forthcoming conditions:

C1) Microrobots operate in close vicinity: $\left(e_{1}, e_{2}\right) \neq(0,0)$.

C2) Microrobots are different: $a_{1} \neq a_{2}$ or $\alpha_{1} \neq \alpha_{2}$.

C3) Function $f(t)$ is not solution of the differential equation:

$$
5 \chi_{2} \chi_{3} \dot{f}(t) f^{4}(t)-\chi_{2} \chi_{4} f^{5}(t)+4 \chi_{1} \chi_{3}^{2}=0
$$

Proof.

C1) When robots are far enough from each other, their magnetic interaction is negligible so that we can set $e_{1}=e_{2}=0$ and the $\left(\mathcal{S}_{x}\right)$ controllability matrix is

$$
\Gamma=\left[\begin{array}{cccc}
0 & \alpha_{1} & -\alpha_{1} a_{1} & \alpha_{1} a_{1}^{2} \\
0 & \alpha_{2} & -\alpha_{2} a_{2} & \alpha_{2} a_{2}^{2} \\
\alpha_{1} & -\alpha_{1} a_{1} & \alpha_{1} a_{1}^{2} & -\alpha_{1} a_{1}^{3} \\
\alpha_{2} & -\alpha_{2} a_{2} & \alpha_{2} a_{2}^{2} & -\alpha_{2} a_{2}^{3}
\end{array}\right]
$$

So $\operatorname{rank}(\Gamma)=3<4$ : three modes are controllable, and another one is associated with a null eigenvalue. The system is consequently simply stable.

Using a state feedback $u=-K x$ leads to closed-loop dynamics $\dot{x}=\left(A_{x}-B_{x} K\right) x$, where the poles can be chosen in $\left\{0, \lambda_{1}, \lambda_{2}, \lambda_{3}\right\}$ with the $\lambda_{i}$ having strictly negative real parts. So there exists an invertible matrix $T$ such that the matrix $D=T^{-1}\left(A_{x}-B_{x} K\right) T$ is a block diagonal matrix whose e.g. first row entries are null. From (8), $T D=\left(A_{x}-B_{x} K\right) T$ first two entries of the first column are given by $T_{31}=T_{41}=0$, and the $T$ invertibility thus requires that $T_{11}^{2}+T_{21}^{2} \neq 0$.

Let $z=T^{-1} x$, then it is straightforward that $\dot{z}=D z$, so that the asymptotic behavior of $z$ is given by:

$$
\begin{cases}z_{1}(t) & =z_{1}(0), \forall t \\ \lim _{t \rightarrow \infty} z_{i}(t) & =0, \forall i \geq 2\end{cases}
$$

Using $x=T z$ in (11), we conclude that

$$
\lim _{t \rightarrow \infty} x(t)=\left[\begin{array}{c}
T_{11} \\
T_{21} \\
0_{2,1}
\end{array}\right] \underbrace{\left[\begin{array}{llll}
1 & 0 & 0 & 0
\end{array}\right] T^{-1} x(0)}_{z_{1}(0)}
$$

C2) The controllability matrix of $\left(\mathcal{S}_{x}\right)$ is given by $\Gamma(t)=$ $\left[\begin{array}{lll}\Gamma_{0}(t) & \ldots & \Gamma_{3}(t)\end{array}\right]$ with $\Gamma_{0}(t)=B_{x}(t)$ and $\Gamma_{i+1}(t)=$
$A_{x}(t) \Gamma_{i}(t)-\dot{\Gamma}_{i}(t)$ [29]. The controllability matrix $\Gamma(t)$ first two columns $\Gamma_{0}$ and $\Gamma_{1}$ are the same as in (10), and the last two columns are replaced by:

$$
\left[\begin{array}{cc}
-\alpha_{1} a_{1} & \alpha_{1} a_{1}^{2}+\frac{4 e_{1} \chi_{3}}{f^{5}(t)} \\
-\alpha_{2} a_{2} & \alpha_{2} a_{2}^{2}-\frac{4 e_{2} \chi_{3}}{f^{5}(t)} \\
\alpha_{1} a_{1}^{2}+\frac{4 e_{1} \chi_{3}}{f^{5}(t)} & 4 e_{1}\left(\frac{5 \chi_{3} \dot{f}(t)}{f^{6}(t)}-\frac{\left(2 a_{1}+a_{2}\right) \chi_{3}+\chi_{4}}{f^{5}(t)}\right)-\alpha_{1} a_{1}^{3} \\
\alpha_{2} a_{2}^{2}-\frac{4 e_{2} \chi_{3}}{f^{5}(t)} & 4 e_{2}\left(\frac{\left(a_{1}+2 a_{2}\right) \chi_{3}+\chi_{4}}{f^{5}(t)}-\frac{5 \chi_{3} \dot{f}(t)}{f^{6}(t)}\right)-\alpha_{2} a_{2}^{3}
\end{array}\right]
$$

Therefore, the determinant of $\Gamma(t)$ is

$$
|\Gamma(t)|=-\frac{4 \chi_{1}}{f^{10}}\left(4 \chi_{1} \chi_{3}^{2}-\chi_{2} \chi_{4} f^{5}+5 \chi_{2} \chi_{3} \dot{f} f^{4}\right)
$$

If $a_{1}=a_{2}$ and $\alpha_{1}=\alpha_{2}$, then $\chi_{3}=\chi_{4}=0$ and it is easy to check that $|\Gamma(t)|=0$, hence we deduce that $\left(\mathcal{S}_{x}\right)$ is not controllable.

C3) If $f(t)$ is solution of (9) then, from (13), it is straightforward that $|\Gamma(t)|=0$ and $\left(\mathcal{S}_{x}\right)$ is not controllable.

Remark 1. (C1) is a necessary condition for the controllability of $\left(\mathcal{S}_{N L}\right)$ since the system is linear for $e_{1}=e_{2}=0$. So it is required that the microrobots operate in close vicinity so that they interact through magnetic interaction. Otherwise $\left(\mathcal{S}_{N L}\right)$ is not controllable yet simply stabilizable on a line depending on the initial condition $x(0)$, see (12). In other words, the velocities $x_{3}, x_{4}$ as well as the linear combination $T_{21} x_{1}-T_{11} x_{2}$ are controllable, whilst $T_{11} x_{1}+T_{21} x_{2}$ is not.

Remark 2. Quite the contrary, $(\boldsymbol{C} 2)$ and $(\boldsymbol{C} 3)$ are related to $\left(\mathcal{S}_{x}\right)$ and thus are not necessary conditions: the nonlinear system $\left(\mathcal{S}_{N L}\right)$ may still be controllable in the case where neither (C2) nor (C3) are satisfied. See e.g. [19], [20], [30] for more details about the controllability of nonlinear systems.

Remark 3. Condition (C2) might seem hard to fulfill because of the magnetic microrobots synthesis normalized processes. Yet it is possible to either coat or aggregate the same magnetic material into different-sized microrobots, e.g. by sphere packing, so that $a_{i} \neq a_{j}$, or use different magnetic over payload ratios to get $\alpha_{i} \neq \alpha_{j}$.

Remark 4. Condition (C3) shows that the controllability of the linearized system $\left(\mathcal{S}_{x}\right)$ depends on the choice of the function $f(t)$ : the system is not controllable if $f(t)$ is solution of (9), however if $\chi_{3}=0$ (i.e. the two robots have the same magnetic sensitivity $\alpha)$ and $a_{1} \neq a_{2}$ the linear system is controllable for all $f(t) \in \mathcal{F} \subset \mathbb{R} \backslash\{0\}$.

\section{TRAJectory PlanNing}

Differential flatness is a powerful tool for planning trajectories, expecially for constrained systems. Yet system (6) is flat only for special cases of $a_{i}$ and $\alpha_{i}$ parameters values; thence we provide another trajectory planning approach encompassing all possible sets of parameters $a_{i}$ and $\alpha_{i}$.

Lemma 1. Admissible reference trajectory for each microrobot $(i)$ is solution of the differential equation

$$
\chi_{3} \ddot{x}_{r i}-\chi_{4} \dot{x}_{r i}=\alpha_{i} \ddot{f}+\alpha_{i} a_{j} \dot{f}+\chi_{1} f^{-4}, j \neq i
$$




$$
\begin{aligned}
& q_{11}(t)=\frac{5\left(8 \chi_{1} \chi_{3}^{2} \dot{f}+6 \chi_{2} \chi_{3} f^{4} \dot{f}^{2}-\chi_{2} \chi_{3} f^{5} \ddot{f}-\chi_{2} \chi_{4} f^{5} \dot{f}\right)}{f\left(5 \chi_{2} \chi_{3} f^{4} \dot{f}+4 \chi_{1} \chi_{3}^{2}-\chi_{2} \chi_{4} f^{5}\right)} \quad q_{42}(t)=\frac{4\left(a_{1} e_{2}+a_{2} e_{1}\right)}{f^{5}} \\
& q_{21}(t)=-\frac{20 \chi_{1} \chi_{3}\left(4 \chi_{3} \dot{f}^{2}+\chi_{3} f \ddot{f}-\chi_{4} f \dot{f}\right)}{f^{2}\left(5 \chi_{2} \chi_{3} f^{4} \dot{f}+4 \chi_{1} \chi_{3}^{2}-\chi_{2} \chi_{4} f^{5}\right)} \quad q_{43}(t)=-a_{1} a_{2}+\frac{4\left(e_{1}+e_{2}\right)}{f^{5}} \\
& q_{31}(t)=-\frac{20 \chi_{1} \chi_{4}\left(4 \chi_{3} \dot{f}^{2}+\chi_{3} f \ddot{f}-\chi_{4} f \dot{f}\right)}{f^{2}\left(5 \chi_{2} \chi_{3} f^{4} \dot{f}+4 \chi_{1} \chi_{3}^{2}-\chi_{2} \chi_{4} f^{5}\right)} \quad q_{44}(t)=-\left(a_{1}+a_{2}\right) \\
& q_{41}(t)=-\frac{20 \chi_{1}\left(\chi_{4}^{2} f^{5} \ddot{f}-4\left(a_{1}-a_{2}\right) \chi_{1} \chi_{3} \dot{f}+f^{5} \dot{f}\left(\left(a_{1}+a_{2}\right) \chi_{4}^{2}+a_{1} a_{2} \chi_{3} \chi_{4}\right)+\left(4 \chi_{4}^{2}-5\left(a_{1}-a_{2}\right) \chi_{2}\right) f^{4} \dot{f}^{2}\right)}{f^{6}\left(5 \chi_{2} \chi_{3} f^{4} \dot{f}+4 \chi_{1} \chi_{3}^{2}-\chi_{2} \chi_{4} f^{5}\right)}
\end{aligned}
$$

and the associated reference control input $u_{r}(t)$ is given by:

$$
u_{r}=\frac{2 \ddot{x}_{r 1}+\left(a_{1}+a_{2}\right) \dot{x}_{r 1}+\left(e_{1}-e_{2}\right) f^{-4}-\ddot{f}-a_{2} \dot{f}}{\alpha_{1}+\alpha_{2}}
$$

Hence the solutions of (15) for a given $f(t)$ gap function are

1) If $\chi_{3} \neq 0$, for $i, j \in\{1 ; 2\}, j \neq i$, we get

$$
x_{r i}(t)=x_{r i}(0)+\dot{x}_{r i}(0) \frac{\chi_{3}}{\chi_{4}}\left(e^{\frac{\chi_{4}}{\chi_{3}} t}-1\right)+\frac{c_{1 i}+c_{2 i}}{\chi_{4}}(t)
$$

with functions

$$
\begin{aligned}
& c_{1 i}(t)=\int_{0}^{t}\left(\alpha_{i} \ddot{f}(\tau)+\alpha_{i} a_{j} \dot{f}(\tau)+\frac{\chi_{1}}{f^{4}(\tau)}\right) e^{\frac{\chi_{4}}{\chi_{3}}(t-\tau)} \mathrm{d} \tau \\
& c_{2 i}(t)=-\int_{0}^{t}\left(\alpha_{i} \ddot{f}(\tau)+\alpha_{i} a_{j} \dot{f}(\tau)+\frac{\chi_{1}}{f^{4}(\tau)}\right) \mathrm{d} \tau
\end{aligned}
$$

2) If $\chi_{3}=0$, then $x_{r i}(t)=x_{r i}(0)+\frac{c_{2 i}(t)}{\chi_{4}}$.

Proof. Eliminating $u_{r}$ from (6) leads to (15) and using $f(t)=$ $x_{r 1}-x_{r 2}$ leads to (16). Integration procedure depends on the order of (15), and thus on $\chi_{3}$. If $\chi_{3}=0$, a straightforward integration leads to the last relationship; otherwise using e.g. the variation of parameters method leads to (17).

\section{LOCALly LyapunOV STABILIZING CONTROLLER}

We proceed using a two steps procedure. We first establish the following lemma, which is useful to change $\left(\mathcal{S}_{x}\right)$ into the forthcoming $\left(\mathcal{S}_{z}\right)$ system on a triangular form, and then synthesize a state feedback control law using the backstepping approach [31] on the triangular system $\left(\mathcal{S}_{z}\right)$.

Lemma 2. Under the conditions of Proposition 1, $\left(\mathcal{S}_{x}\right)$ is controllable and the mapping

$$
\Phi:\left(\begin{array}{c}
x(t) \\
t
\end{array}\right) \longmapsto\left(\begin{array}{c}
z(t) \\
t
\end{array}\right)=\left(\begin{array}{c}
T_{z}(t) x(t) \\
t
\end{array}\right)
$$

with the matrix

$$
T_{z}^{-1}(t)=\left[\begin{array}{cccc}
-\frac{4 \chi_{1}}{f^{5}(t)} & \alpha_{1} a_{2} & \alpha_{1} & 0 \\
-\frac{4 \chi_{1}}{f^{5}(t)} & \alpha_{2} a_{1} & \alpha_{2} & 0 \\
-\frac{20 \chi_{1} \dot{f}(t)}{f^{6}(t)} & -\frac{4 \chi_{1}}{f^{5}(t)} & \alpha_{1} a_{2} & \alpha_{1} \\
-\frac{20 \chi_{1} \dot{f}(t)}{f^{6}(t)} & -\frac{4 \chi_{1}}{f^{5}(t)} & \alpha_{2} a_{1} & \alpha_{2}
\end{array}\right]
$$

is a diffeomorphism which transforms $\left(\mathcal{S}_{x}\right)$ into the LTV triangular system $\left(\mathcal{S}_{z}\right)$ :

$$
\left(\mathcal{S}_{z}\right): \dot{z}(t)=A_{z}(t) z(t)+B_{z} u(t)
$$

With the $q_{i j}(t)$ given by (14) and matrices $A_{z}(t)$ and $B_{z}$ are:

$$
A_{z}(t) \mid B_{z}=\left[\begin{array}{llll|l}
q_{11}(t) & 1 & 0 & 0 & 0 \\
q_{21}(t) & 0 & 1 & 0 & 0 \\
q_{31}(t) & 0 & 0 & 1 & 0 \\
q_{41}(t) & q_{42}(t) & q_{43}(t) & q_{44}(t) & 1
\end{array}\right]
$$

Proof. Using (19), we get

$$
\left|T_{z}^{-1}(t)\right|=-\frac{4 \chi_{1}\left(4 \chi_{1} \chi_{3}^{2}-\chi_{2} \chi_{4} f^{5}(t)+5 \chi_{2} \chi_{3} \dot{f}(t) f^{4}(t)\right)}{f^{10}(t)}
$$

From Proposition 1, it is straightforward that $T_{z}^{-1}(t)$ is invertible for all $t$, and it follows that $\Phi$ is a diffeomorphism.

Along the reference trajectory, one has (7). Since $z=$ $T_{z}(t) x$, it follows that $z$ is solution of (20) with:

$$
\left\{\begin{array}{l}
A_{z}(t)=T_{z}(t) A_{x}(t) T_{z}^{-1}(t)+\dot{T}_{z}(t) T_{z}^{-1}(t) \\
B_{z}=T_{z}(t) B_{x}
\end{array}\right.
$$

whose computation gives the triangular matrice (21) with the $q_{i j}(t)$ given by (14).

Assumption 1. The system full-state is available for measurement.

In practice, this assumption is hardly fulfilled since medical imagers provide the robots positions at best. Worst still, susceptibility artifacts may jeopardize the localization of both robots, so that it is likely that only a single information about these positions is accessible. Linear state observers have been recently developed [22], [32] assuming that each robot position is accessible and neglecting the magnetic interaction. Nonlinear observers have been proposed in [14], [16] but for a single agent. Observation and output feedback synthesis have to be addressed prior to dispensing with Assumption 1 .

Proposition 2. Provided that the conditions of the Assumption 1 and Proposition 1 are satisfied, let $\left(x_{r}, u_{r}\right)(t)$ denote any admissible trajectory and its reference control input given by Lemma 1. The control law $u=\psi\left(z(t), z_{r}(t)\right)$ given by

$$
\psi\left(z(t), z_{r}(t)\right)=\sum_{i=1}^{4} \delta_{i}(t)\left(z_{i}(t)-z_{r i}(t)\right)+u_{r}(t)
$$

with $z_{r}=T_{z} x_{r}, \delta_{i}$ and $u_{r}$ respectively given by (22) for $k_{i}>0$ and (16), guarantees the Lyapunov global stability of system $\left(\mathcal{S}_{x}\right)$-and thence the local stability of system $\left(\mathcal{S}_{N L}\right)$ along the trajectory $x_{r}(t)$. 


$$
\begin{aligned}
\delta_{4}= & -\left[\sum_{i=1}^{4} k_{i}+q_{11}+q_{44}\right], \quad \delta_{3}=-\left[\left(\sum_{i=1}^{4} k_{i}\right) q_{11}+\left(\sum_{i=1}^{4} \sum_{j=i+1}^{4} k_{i} k_{j}\right)+q_{11}^{2}+q_{21}+3 \dot{q}_{11}+q_{43}+3\right] \\
\delta_{2}= & -\left[\left(\sum_{i=1}^{4} k_{i}\right)\left(1+q_{11}^{2}+2 \dot{q}_{11}+q_{21}\right)+\left(\sum_{i=1}^{4} \sum_{j=i+1}^{4} k_{i} k_{j}\right) q_{11}+q_{11}^{3}+3 q_{11}+3 \ddot{q}_{11}+2 \dot{q}_{21}+2 q_{11} q_{21}\right. \\
& \left.+5 q_{11} \dot{q}_{11}+k_{1}+k_{4}+q_{31}+q_{42}+\left(\sum_{i=1}^{4} \sum_{j=i+1}^{4} \sum_{h=j+1}^{4} k_{i} k_{j} k_{h}\right)\right] \\
\delta_{1}= & -\left[\left(\sum_{i=1}^{4} k_{i}\right)\left(q_{11}^{3}+\dot{q}_{21}+q_{11}+\ddot{q}_{11}+3 q_{11} \dot{q}_{11}+2 q_{11} q_{21}+q_{31}\right)+\left(\sum_{i=1}^{4} \sum_{j=i+1}^{4} k_{i} k_{j}\right)\left(q_{11}^{2}+q_{21}+\dot{q}_{11}\right)\right. \\
& +\left(q_{11}^{3}+3 q_{11}+3 \dot{q}_{21}+2 q_{31}+4 \ddot{q}_{11}+6 q_{11} \dot{q}_{11}+3 q_{11} q_{21}+k_{1}+k_{4}+\sum_{i=1}^{4} \sum_{j=i+1}^{4} \sum_{h=j+1}^{4} k_{i} k_{j} k_{h}\right) q_{11} \\
& \left.+q_{21}^{2}+3 q_{21}+\dddot{q}_{11}+3 \dot{q}_{11}^{2}+3 \dot{q}_{11}+\ddot{q}_{21}+\dot{q}_{31}+4 q_{21} \dot{q}_{11}+q_{41}+1+k_{1} k_{2}+k_{1} k_{4}+k_{3} k_{4}+\prod_{i=1}^{4} k_{i}\right]
\end{aligned}
$$

Proof. Let $y_{i}=z_{i}-z_{r i}, \bar{z}_{1}=y_{1}$ and $\bar{z}_{j}=y_{j}-z_{d j}$ for some stabilizing functions $z_{d j}, j=2,3,4$.

- Step 1: Differentiating a candidate Lyapunov function $V_{1}=\frac{1}{2} \bar{z}_{1}^{2}$, we obtain

$$
\dot{V}_{1}=\bar{z}_{1}\left(y_{2}+q_{11} \bar{z}_{1}\right)=\bar{z}_{1}\left(\bar{z}_{2}+q_{11} \bar{z}_{1}+z_{d 2}\right)
$$

Taking $z_{d 2}=-\left(k_{1}+q_{11}\right) \bar{z}_{1}$ gives $\dot{V}_{1}=-k_{1} \bar{z}_{1}^{2}+\bar{z}_{1} \bar{z}_{2}$. Since $\dot{\bar{z}}_{1}=y_{2}+q_{11} \bar{z}_{1}=\bar{z}_{2}-k_{1} \bar{z}_{1}$, we get

$$
\dot{\bar{z}}_{2}=y_{3}+\left(k_{1}+q_{11}\right) \bar{z}_{2}-\left[k_{1}\left(k_{1}+q_{11}\right)-\dot{q}_{11}-q_{21}\right] \bar{z}_{1}
$$

- Step 2: Let $V_{2}=\frac{1}{2} \sum_{i=1}^{2} \bar{z}_{i}^{2}=V_{1}+\frac{\bar{z}_{2}^{2}}{2}$, then

$$
\begin{aligned}
\dot{V}_{2}= & -k_{1} \bar{z}_{1}^{2}+\bar{z}_{2}\left[\bar{z}_{3}+z_{d 3}+\left(k_{1}+q_{11}\right) \bar{z}_{2}\right. \\
& \left.-\left(k_{1}\left(k_{1}+q_{11}\right)-\dot{q}_{11}-q_{21}-1\right) \bar{z}_{1}\right]
\end{aligned}
$$

The choice $z_{d 3}=-\left(\sum_{i=1}^{2} k_{i}+q_{11}\right) \bar{z}_{2}+\left[k_{1}\left(k_{1}+q_{11}\right)-\right.$ $\left.\dot{q}_{11}-q_{21}-1\right] \bar{z}_{1}$ leads to $\dot{V}_{2}=-\sum_{i=1}^{2} k_{i} \bar{z}_{i}^{2}+\bar{z}_{2} \bar{z}_{3}$. Since $\dot{\bar{z}}_{2}=\bar{z}_{3}-k_{2} \bar{z}_{2}-\bar{z}_{1}$, then $\dot{\bar{z}}_{3}=y_{4}+q_{31} \bar{z}_{1}-\dot{z}_{d 3}$, i.e.

$$
\begin{aligned}
\dot{\bar{z}}_{3}= & \bar{z}_{4}+z_{d 4}+\left[\sum_{i=1}^{2} k_{i}+q_{11}\right] \bar{z}_{3}-\left[\sum_{i=1}^{2} k_{i}^{2}\right. \\
& \left.+k_{1} k_{2}-1+q_{11} \sum_{i=1}^{2} k_{i}-2 \dot{q}_{11}-q_{21}\right] \bar{z}_{2} \\
& -\left[\sum_{i=1}^{2}(3-i) k_{i}-k_{1}^{3}+\left(1-k_{1}^{2}\right) q_{11}\right. \\
& \left.+2 k_{1} \dot{q}_{11}-\ddot{q}_{11}+k_{1} q_{21}-\dot{q}_{21}-q_{31}\right] \bar{z}_{1}
\end{aligned}
$$

- Step 3: Differentiating $V_{3}=\frac{1}{2} \sum_{i=1}^{3} \bar{z}_{i}^{2}=V_{2}+\frac{\bar{z}_{3}^{2}}{2}$ gives $\dot{V}_{3}=-\sum_{i=1}^{2} k_{i} \bar{z}_{i}^{2}+\bar{z}_{3}\left(\dot{\bar{z}}_{3}+\bar{z}_{2}\right)$. So the choice

$$
\begin{aligned}
z_{d 4}= & -\left(\sum_{i=1}^{3} k_{i}+q_{11}\right) \bar{z}_{3}+\left[\sum_{i=1}^{2} k_{i}^{2}+k_{1} k_{2}-2\right. \\
& \left.+\left(\sum_{i=1}^{2} k_{i}\right) q_{11}-2 \dot{q}_{11}-q_{21}\right] \bar{z}_{2} \\
& +\left[\sum_{i=1}^{2}(3-i) k_{i}-k_{1}^{3}+\left(1-k_{1}^{2}\right) q_{11}\right. \\
& \left.+2 k_{1} \dot{q}_{11}-\ddot{q}_{11}+k_{1} q_{21}-\dot{q}_{21}-q_{31}\right] \bar{z}_{1}
\end{aligned}
$$

TABLE I

PARAMETERS VALUeS

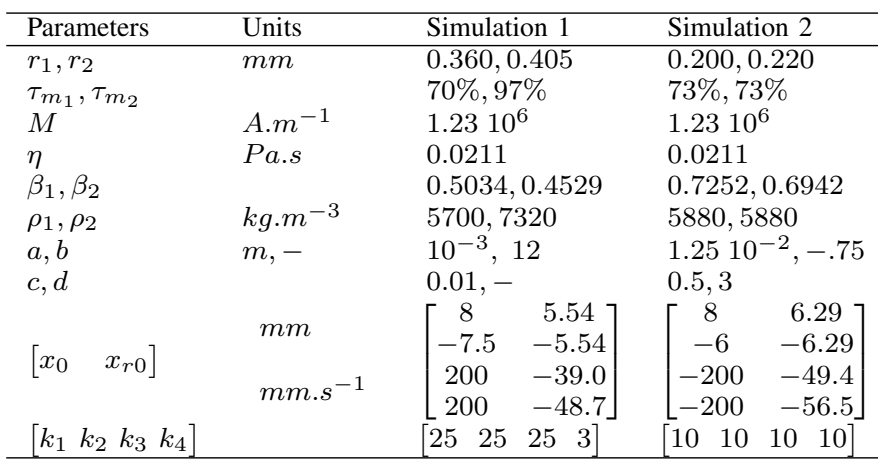

\section{Simulations}

In each simulation, the reference trajectory for both microrobots are given by either Lemma 1.1 or Lemma 1.2, depending on the $\chi_{3}$ value, from any non vanishing function 
$f(t)^{3}$. The control law $u=\psi\left(z(t), z_{r}(t)\right)$ is computed using (23) with gains $k_{i}$, parameters and initial conditions given in Table I for microrobots composed of $\tau_{m} \mathrm{NdFeB} 35$ $\left(\rho=7500 \mathrm{~kg} \cdot \mathrm{m}^{-3}, M=1.2310^{6} \mathrm{~A} \cdot \mathrm{m}^{-1}\right)$ and $\left(1-\tau_{m}\right)$ payload $\left(\rho=1500 \mathrm{~kg} \cdot \mathrm{m}^{-3}\right)$ navigating in a small artery of radius $1.5 \mathrm{~mm}$. Parameters $\beta$ are computed using [27]. In order to avoid actuator damage, the control input is timescaled as $u_{a}(t)=u(t) / k(t)$ with $k(t)=\max \left(1,|u(t)| / u_{\text {sat }}\right)$ with $u_{\text {sat }}=0.2$ T.m $\mathrm{m}^{-1}$. The state is affected by an additive Gaussian white noise with a $0.1 \mathrm{~mm}$ standard deviation.

\section{A. First Simulation}

Simulation 1, shown in the left column of Figure 2, illustrates the case where $\chi_{3} \neq 0$, i.e. the microrobots do not have the same magnetic sensitivity $\alpha$. We choose a function $f(t)=a\left(b-e^{c \frac{\chi_{4}}{\chi_{3}} t}\right)$ where $a, b \in \mathbb{R}^{*}$, and $c: c^{-1} \in \mathbb{N}^{*}$. This simulation illustrates a leader-follower strategy since the second robot, mainly composed of magnetic material with a small payload capacity, can push the first robot embedding more payload.

Figure 2(a) illustrates the reference distance $f(t)=x_{r 1}(t)-$ $x_{r 2}(t)$ (red dashed line) and the real one $x_{1}(t)-x_{2}(t)$ (black solid line) between microrobots (1) and (2). The Fig.2(c) (resp. Fig.2(e)) illustrates the reference and real positions of robots (1) and (2) (resp. their velocities). These two figures show that the real positions and velocities converge to the reference ones within 2.5 seconds. The robots positions and velocities have an overshoot before converging, i.e. the system trajectory initially tends to diverge. The reason for such a behavior is twofold: the control input briefly reaches the upper saturation $\left(u=0.2 \mathrm{~T} . \mathrm{m}^{-1}\right)$, as illustrated by Fig. $2(\mathrm{~g})$, and -most importantly- the initial state is far from the reference one. So the linear approximation of the nonlinear term $g(x)=\left(x_{1}-x_{2}\right)^{-4}$ from (6) along the reference trajectory induces an offset in the compensation of this term in the controller since the initial error is important (around 75\% on the initial $g$ value). Simulation 1 shows that the trajectory $\left(x_{i}, \dot{x}_{i}\right)$ of each microrobot $(i)$ converges to the reference trajectory $\left(x_{r i}, \dot{x}_{r i}\right)$ despite the saturation of the control input, and illustrates the closed-loop system local stability.

\section{B. Second Simulation}

Simulation 2 illustrates the case $\chi_{3}=0$, i.e. the two robots have the same magnetic sensitivity $\alpha_{1}=\alpha_{2}$, and the choice of an oscillating reference relative distance $f(t)=a(1+$ $\left.b \sin (d t) e^{-c t}\right)^{-1 / 4}$ between the two robots. This simulation illustrates a different strategy using quite similar microrobots.

The reference and real values of the distance between the microrobots are plotted on Fig. 2(b): the convergence occurs after a $3 s$ transient phase, as well as the two robots positions convergence to their reference $x_{r i}$ depicted in Fig. 2(d). The microrobots real and reference positions (resp. velocities) are depicted in Fig. 2(d) (resp. Fig. 2(f)). We observe the same initial behavior than in the first simulation, for the same

\footnotetext{
${ }^{3}$ In the present simulations, such functions are chosen in order both to simplify the computations in Lemma 1 and to ensure that (C3) is fulfilled.
}

reasons but with dominating saturation events within the first $0.5 \mathrm{~s}$. Indeed the control input, depicted in Fig. 2(h), exhibits upper and lower saturation events at the simulation beginning: the convergence is a bit longer than in the first simulation. The noise does not affect significantly the microrobots positions tracking, contrary to the velocity tracking. The interrobots distance error reaches a peak of $4.5 \mathrm{~mm}$ during the saturation events, as can be seen on Fig. 2(b), inducing a $300 \%$ error on the estimation of $g$, which illustrates the limits of robustness of the linearization approach.

TABLE II

Tracking ERrors StANDARd DEVIATIONS FOR $t \in[3,5] s$

\begin{tabular}{clcc}
\hline Parameters & Units & Simulation 1 & Simulation 2 \\
\hline$\sigma_{\tilde{x}_{1}}$ & $m m$ & 0.008 & 0.106 \\
$\sigma_{\tilde{x}_{2}}$ & $m m$ & 0.010 & 0.121 \\
$\sigma_{\tilde{x}_{3}}$ & $m m . s^{-1}$ & 0.060 & 0.668 \\
$\sigma_{\tilde{x}_{4}}$ & $m m . s^{-1}$ & 0.086 & 0.773 \\
\hline
\end{tabular}

The tracking standard deviations are given in Table II once the convergence is achieved in either simulation. In both simulations, the gains have been chosen so that the position tracking performs better than the velocity tracking in order to lessen the effects of the nonlinear term $g$. The parameter $\chi_{1}$ differs by a factor ten so the standard deviations differ roughly by one order of magnitude. Indeed, this parameter affects the diffeomorphism mapping $\left(\mathcal{S}_{x}\right)$ into $\left(\mathcal{S}_{z}\right)$ so that the $z$-state is ten times more sensitive to the output noise in Simulation 2.

\section{DISCUSSION}

This paper first addresses the local controllability of a twoagent microrobotic underactuated system, providing a necessary condition -microrobots must operate in close vicinityand sufficient conditions impacting the microagents design and trajectory planning. The results are partial since based on the linearized LTV system, not on the nonlinear initial system, yet it is the first controllability result for a multi-agent microrobotic underactuated system. Trajectory planning was addressed in [33] within a fully actuated framework; we have addressed it in the underactuated case. In the end, we have proven the Lyapunov local stability of the system along any admissible trajectory.

Contrary to most previous studies that considered independent control, we have considered the system underactuation inherent to a number of magnetic platforms like MRI devices, as in [21] but addressing directly underactuation. Using a single control input, a scalar (the relative distance) is stabilized in [25], both robots positions are stabilized in [21], whilst our methodology guarantees the stabilization of both positions and velocities of the two agents, thus enabling trajectory control unlike the aforementioned works. However the linear analysis reaches its limits when dealing with such a nonlinear system, and on-going nonlinear controllability analysis and control synthesis may improve the present results. For instance, simulations show that the linearization of $\left(\mathcal{S}_{N L}\right)$ may induce important tracking issues when the microrobots are initially far away from the reference; working directly on the nonlinear system may avoid such issues. 


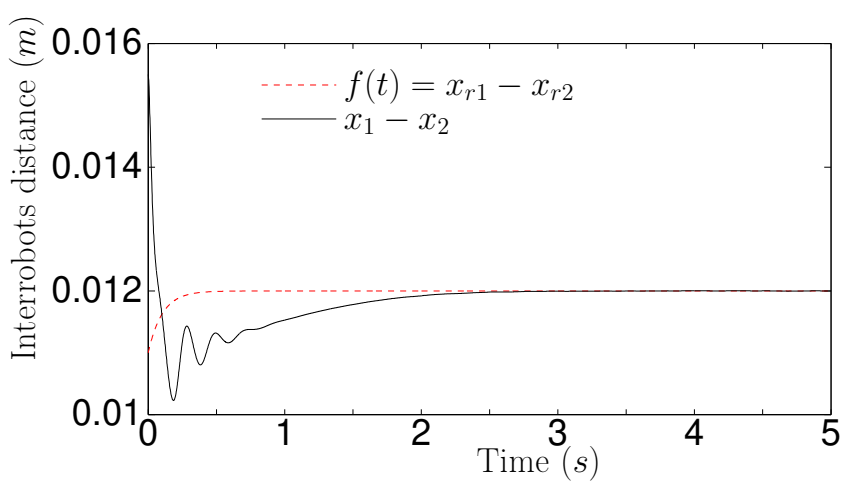

(a) Distance between the two microrobots

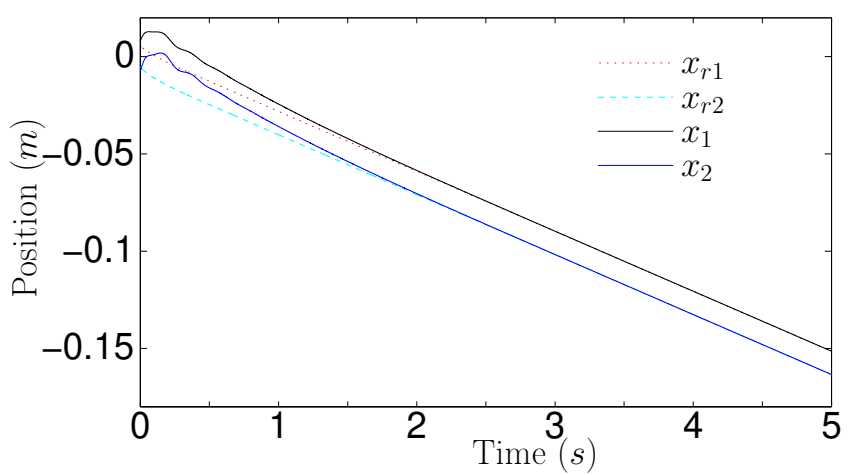

(c) Microrobots positions

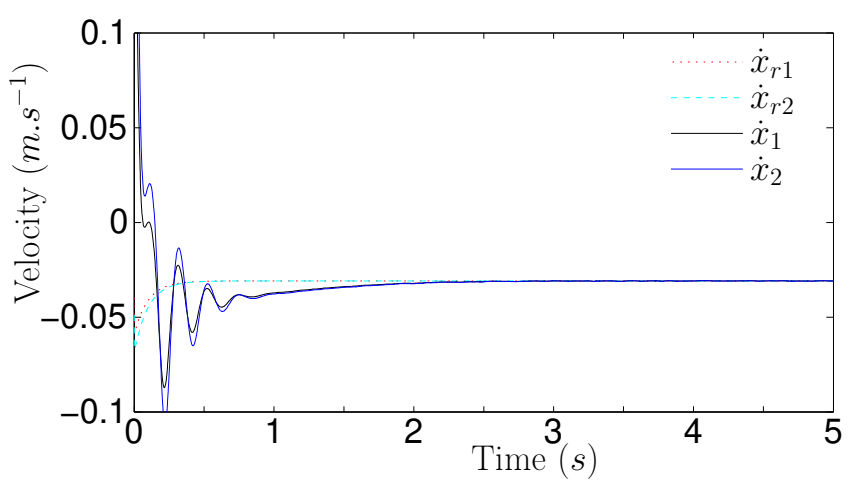

(e) Microrobots velocities

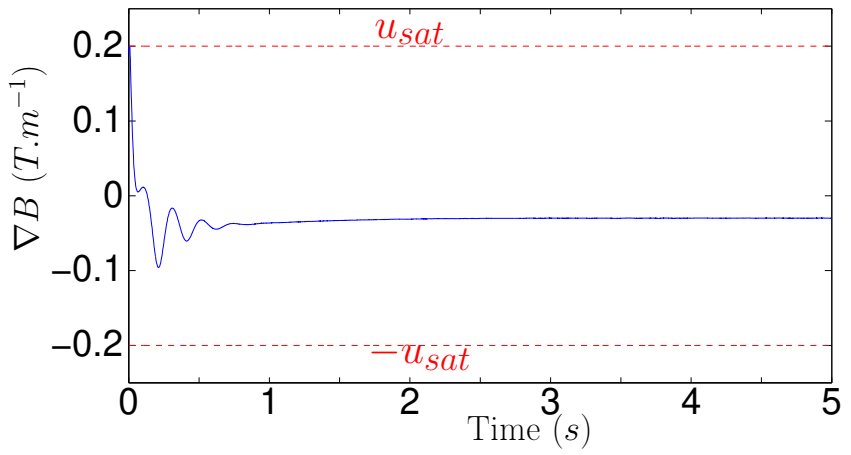

(g) Control input

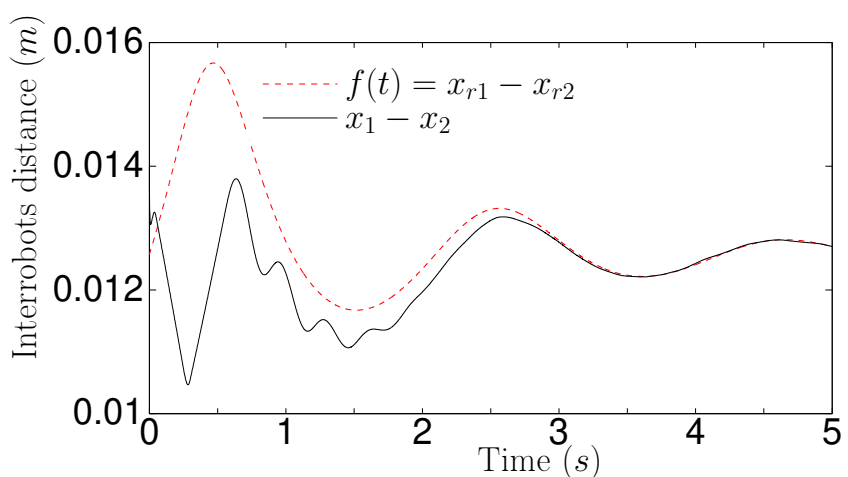

(b) Distance between the two microrobots

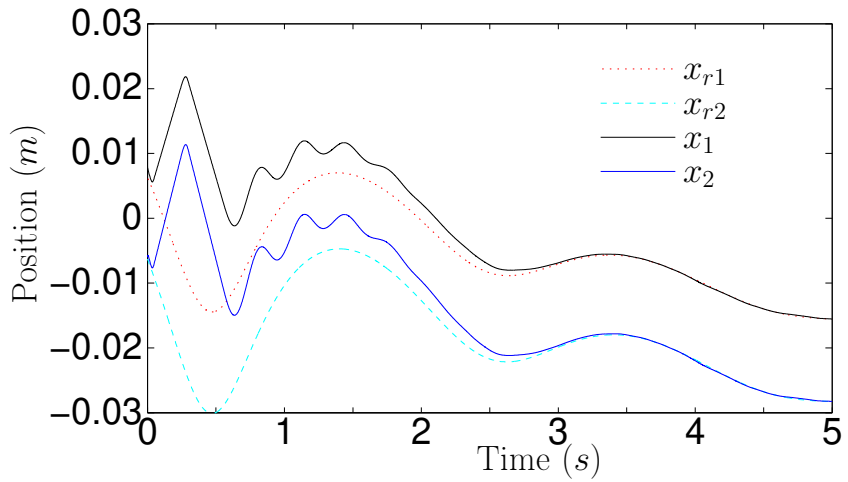

(d) Microrobots positions

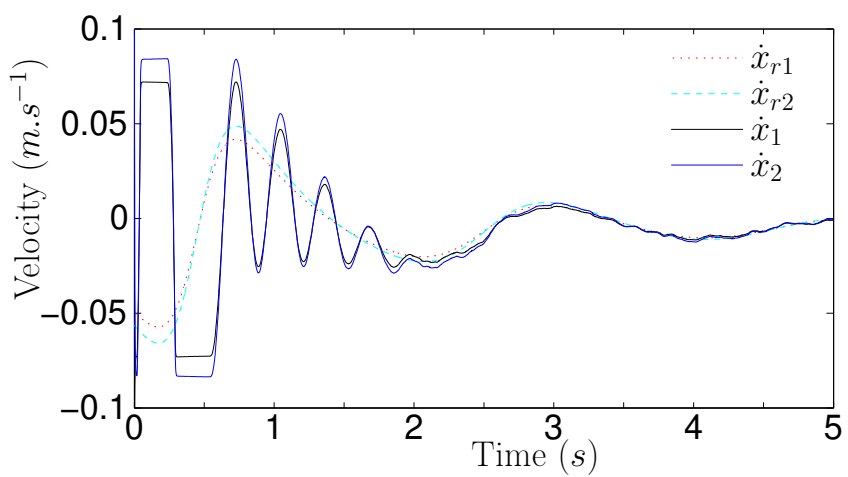

(f) Microrobots velocities

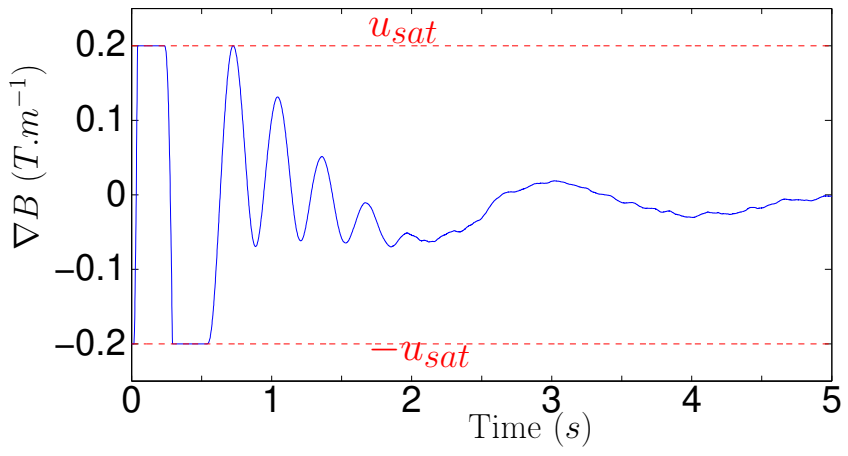

(h) Control input

Fig. 2. Distance between the two microrobots, microrobots positions and velocities, and control input. From left to right: Simulation 1 and 2 . From top to bottom: (a)-(b) distance between the microrobots (1) and (2), (c)-(d) microrobots positions, (e)-(f) microrobots velocities and (g)-(h) control inputs. On subfigures (a)- (b), real and reference distance are depicted by solid black and red dashed lines respectively, (c)-(d) real and reference positions of the microrobots (1) and (2) are depicted by solid black, red dotted and solid blue, cyan dashed lines respectively, (e)-(f) real and reference velocities of the microrobots (1) and (2) are depicted by solid black, red dotted and solid blue, cyan dashed lines respectively. 


\section{CONCLUSION}

In this paper, we have synthesized a backstepping state feedback in order to locally Lyapunov stabilize the position and velocity of two magnetic microrobots in a blood vessel using the same single control input. To this end, we have provided a preliminary controllability study of this underactuated drift system, which was lacking in the literature. In particular, we have provided a necessary condition (condition $\mathbf{C} 1$ of Proposition 1): the microrobots must be close enough to exert magnetic interaction in order to be controllable. Trajectory planning has also been addressed as well as the control law synthesis using a backstepping approach.

Further perspectives, which are currently under our investigation, include the extension of the present approach to a higher number of agents to design microrobotic systems more consistent with therapeutic constraints while preserving the system controllability. Besides, biophysical systems are fatally affected by an important variability, so robustness to the induced parametric uncertainties has also to be addressed. Furthermore, magnetic microrobots localized by MRI are well known for producing artifacts whose size is far higher than the agents radius [34], [35], so it is highly speculative to assume that each microrobot position is measurable. Anyway microrobots and blood velocities are also hardly measurable, thus breaking Assumption 1. Whilst nonlinear observers are necessary to propose stabilizing output feedbacks, the observation of multi-microrobot systems has not been addressed except in the fully actuated linear case [22], [32]. Ongoing work is thus led to ensure stability despite these uncertainties and lack of information, an issue that we have already addressed for a single microrobot using either adaptive approach [14] or state estimation in [16] using the same backstepping synthesis.

\section{REFERENCES}

[1] J.-B. Mathieu, G. Beaudoin, and S. Martel, "Method of propulsion of a ferromagnetic core in the cardiovascular system through magnetic gradients generated by an MRI system," IEEE Trans. Bio-Med. Eng., vol. 53, no. 2, pp. 292-299, 2006.

[2] S. Floyd, C. Pawashe, and M. Sitti, "Two-dimensional contact and noncontact micromanipulation in liquid using an untethered mobile magnetic microrobot," IEEE Trans. Robot., vol. 25, no. 6, pp. 13321342, 2009.

[3] D. Frutiger, B. Kratochvil, K. Vollmers, and B. Nelson, "Small, fast, and under control: wireless resonant magnetic micro-agents," Int. J. Robot. Res., vol. 29, no. 5, pp. 613-636, 2010.

[4] A. Servant, F. Qiu, M. Mazza, K. Kostarelos, and B. J. Nelson, "Controlled in vivo swimming of a swarm of bacteria-like microrobotic flagella," Advanced Materials, vol. 27, no. 19, pp. 2981-2988, 2015.

[5] M. C. Lagomarsino, F. Capuani, and C. P. Lowe, "A simulation study of the dynamics of a driven filament in an aristotelian fluid," $J$. Theor. Biol., vol. 224, no. 2, pp. 215-224, 2003.

[6] A. A. Evans and E. Lauga, "Propulsion by passive filaments and active flagella near boundaries," Phys. Rev. E, vol. 82, no. 4, pp. $041915.1-$ 041 915.12, 2010.

[7] R. Dreyfus, J. Beaudry, M. L. Roper, M. Fermigier, H. A. Stone, and J. Bibette, "Microscopic artificial swimmers," Nature, vol. 437, pp. 862865, 2005.

[8] L. Zhang, K. E. Peyer, and B. J. Nelson, "Artificial bacterial flagella for micromanipulation,” Lab Chip, vol. 10, no. 17, pp. 2203-2215, 2010.

[9] J. J. Abbott, K. E. Peyer, M. C. Lagomarsino, L. Zhang, L. X. Dong, I. K. Kaliakatsos, and B. J. Nelson, "How should microrobots swim?" Int. J. Robot. Res., vol. 28, pp. 1434-1447, 2009.

[10] B. J. Nelson, I. K. Kaliakatsos, and J. J. Abbott, "Microrobots for minimally invasive medecine," Ann. Rev. Biomed. Eng., vol. 12, pp. 55-85, 2010.
[11] L. Giraldi and J. B. Pomet, "Local controllability of the two-link magneto-elastic micro-swimmer," IEEE Trans. Autom. Control, vol. 62, no. 5, pp. 2512-2518, 2017.

[12] M. Fruchard, L. Arcese, and E. Courtial, "Estimation of the blood velocity for nanorobotics," IEEE Trans. Robot., vol. 30, no. 1, pp. 93102, 2014.

[13] L. Arcese, M. Fruchard, and A. Ferreira, "Endovascular magneticallyguided robots: navigation modeling and optimization," IEEE Trans. BioMed. Eng., vol. 59, no. 4, pp. 977-987, 2012.

[14] - "Adaptive controller and observer for a magnetic microrobot," IEEE Trans. Robot., vol. 29, no. 4, pp. 1060-1067, 2013.

[15] T. Xu, G. Hwang, N. Andreff, and S. Régnier, "Planar path following of 3D steering scaled-up helical microswimmers," IEEE Trans. Robot., vol. 31 , no. 1 , pp. $117-127,2015$.

[16] L. Sadelli, M. Fruchard, and A. Ferreira, "2D observer-based control of a vascular microrobot," IEEE Trans. Autom. Control, vol. 62, no. 5, pp. 2194-2206, 2017.

[17] P. Pouponneau, J. C. Leroux, G. Souez, L. Gaboury, and S. Martel, “Coencapsulation of magnetic nanoparticles and doxorubicin into biodegradable microcarriers for deep tissue targeting by vascular navigation," Biomaterials, vol. 32, pp. 3481-3486, 2011.

[18] Y. Kantaros, B. V. Johnson, S. Chowdhury, D. J. Cappelleri, and M. M. Zavlanos, "Control of magnetic microrobot teams for temporal micromanipulation tasks," IEEE Trans. Robot., vol. 34, no. 6, pp. 14721489, 2018.

[19] H. J. Sussmann, "A general theorem on local controllability," SIAM J. on Cont. and Opt., vol. 25, no. 1, pp. 158-194, 1987.

[20] M. Kawski, "High-order small-time local controllability," in Nonlinear Controllability and Optimality, H. J. Sussmann, Ed. Dekker, 1987, pp. 431-467.

[21] A. Eqtami and P. E. Dupont, "Stabilizing the relative position of millirobots inside an mri scanner considering magnetic interaction forces," Proc. IEEE/RSJ Int. Conf. Intell. Robot. Syst., pp. 3220-3226, 2015.

[22] A. Denasi and S. Misra, "Independent and leader-follower control for two magnetic micro-agents," IEEE Robot. Autom. Lett., vol. 3, no. 1, pp. 218-225, 2018.

[23] S. Floyd, E. Diller, C. Pawashe, and M. Sitti, "Control methodologies for a heterogeneous group of untethered magnetic micro-robots," Int. J. Robot. Res., vol. 30, no. 13, pp. 1553-1565, 2011.

[24] E. Diller, S. Floyd, C. Pawashe, and M. Sitti, "Control of multiple heterogeneous magnetic microrobots in two dimensions on nonspecialized surfaces," IEEE Trans. Robot., vol. 28, no. 1, pp. 172-182, 2012.

[25] M. Salehizadeh and E. Diller, "Two-agent formation control of magnetic microrobots in two dimensions," J. Microbio Robot., vol. 12, no. 1, pp. 9-19, 2017.

[26] A. Eqtami, O. Felfoul, and P. E. Dupont, "MRI-powered closed-loop control for multiple magnetic capsules," Proc. IEEE/RSJ Int. Conf. Intell. Robot. Syst., pp. 3536-3542, September 2014.

[27] R. Kehlenbeck and R. D. Felice, "Empirical relationships for the terminal settling velocity of spheres in cylindrical columns," Chem. Eng. Technol., vol. 21, pp. 303-308, 1999.

[28] P. Vartholomeos and C. Mavroidis, "In silico studies of magnetic microparticle aggregations in fluid environments for MRI-guided drug delivery," IEEE Trans. Bio-Med. Eng., vol. 59, no. 11, pp. 3028-3038, 2012.

[29] L. M. Silverman and H. E. Meadows, "Controllability and observability in time-variable linear systems," SIAM J. on Cont. and Opt., vol. 5, pp. 64-73, 1967.

[30] J. Basto Gonçalves, "Local controllability along a reference trajectory," J. Math. Anal. Appl., vol. 158, no. 1, pp. 55-62, 1991.

[31] I. Kanellakopoulos, P. Kokotović, and A. Morse, "A toolkit for nonlinear feedback design,” Syst. Control Lett., vol. 18, pp. 83-92, 1992.

[32] L. Yang, Q. Wang, and L. Zhang, "Model-free trajectory tracking control of two-particle magnetic microrobot," IEEE Trans. Nanotechnol., vol. 17, no. 4, pp. 697-700, July 2018.

[33] P. Vartholomeos, M. R. Akhavan-Sharif, and P. E. Dupont, "Motion planning for multiple millimeter-scale magnetic capsules in a fluid environment," Proc. IEEE Int. Conf. Robot. Autom., pp. 1927-1932, May 2012.

[34] O. Felfoul, J.-B. Mathieu, G. Beaudoin, and S. Martel, "In vivo MRtracking based on magnetic signature selective excitation," IEEE Trans. Med. Imag., vol. 27, no. 1, pp. 28-35, 2008.

[35] T. Wortmann, C. Dahmen, and S. Fatikow, "Study of MRI susceptibility artifacts for nanomedical applications," J. Nanotechnol. Eng. Med., vol. 1, no. 4, pp. 041002.1-041 002.5, 2010. 